\title{
OCORRÊNCIA SIMULTÂNEA DE HEMANGIOSSARCOMA SUBCUTÂNEO E QUIMIODECTOMA EM CADELA - RELATO DE CASO
}

Érica Almeida Viscone ${ }^{1}$, Alessandra Castro Rodrigues ${ }^{1}$, Lígia Assunção Oliveira1, Karina Michelle Braga', Márcio de Barros Bandarra².

1- Residente em Patologia Animal, Universidade Federal de Uberlândia, Uberlândia-MG, Brasil. E-mail: ericaviscone@gmail.com

2- Docente da Faculdade de Medicina Veterinária, Universidade Federal de Uberlândia, Uberlândia - MG, Brasil.

Recebido em: 02/10/2017 - Aprovado em: 21/11/2017 - Publicado em: 05/12/2017 DOI: 10.18677/EnciBio_2017B44

Hemangiossarcomas são neoplasias de origem do endotélio vascular ou de células precursoras do endotélio, que pode ocorrer em qualquer parte vascularizada do organismo sendo de ocorrência comum em cães. Quimiodectoma é uma neoplasia de quimiorreceptores que pode se desenvolver nos corpos aórtico e carotídeo, levando a sintomas clínicos de descompensação cardiorrespiratória ou não apresentando nenhum sinal clínico. Esse trabalho teve como objetivo relatar um caso de ocorrência simultânea de hemangiossarcoma subcutâneo e quimiodectoma em uma cadela da raça Boxer, 13 anos, encaminhada para necropsia no setor de Patologia Animal do Hospital Veterinário da Universidade Federal de Uberlândia MG. Macroscopicamente, o animal apresentava uma massa em região de osso frontal, com crescimento progressivo, próximo ao globo ocular direito. $\mathrm{O}$ animal apresentava tremores, incoordenação, fraqueza e perda de peso progressiva. Foi realizada radiografia do crânio onde constatou-se lise óssea. Ainda, massa em corpo aórtico. Ao exame histopatológico, diagnosticou-se hemangiossarcoma na massa da cabeça e quimiodectoma na massa em base do coração.

PALAVRAS - CHAVE: endotélio, neoplasia, quimiorreceptores.

\section{SIMULTANEOUS OCCURRENCE OF SUBCUTANEOUS HEMANGIOSARCOMA AND CHEMODECTOMA IN DOG - CASE REPORT}

\begin{abstract}
Hemangiosarcoma of endotelial origin, metastatic, with aggressive biological behavior, can occur in any vascular site in the body and is common in dogs. Chemodectomas are tumors formed from chemoreceptors present at the heart's base involving the aortic and carotid body, can cause cardiorespiratory failure or be asymptomatic. The aim of these study was report an occurrence of hemangiosarcoma and chemodectoma at same time in a Boxer dog, thirteen years old, wich was necropsied at Animal Pathology Department, Federal University of Uberlândia. Macroscopy, there was at frontal bone mass, progressive growth, near to right eye, the animal had neurologycal signs. At skull radiograph showed osteolysis caused by the mass. Furthermore, was found a mass at aortic body. Histopathologyc diagnosis was hemangiosarcoma, at head, and chemodectoma at heart bases.
\end{abstract}

KEYWORDS: endothelium, chemoreceptors, neoplasia. 


\section{INTRODUÇÃO}

Hemangiossarcoma é uma neoplasia originada tanto de células do endotélio vascular quanto de células precursoras do endotélio, maligna e agressiva (LAMERATO-KOZICKI et al., 2006; YAMAMOTO et al., 2013). Cães são frequentemente acometidos por essa neoplasia (FLORES et al., 2012; GÖRITZ et al., 2013; YAMAMOTO et al., 2013; CHIKAZAWA et al., 2013; SOARES et al., 2017) que pode ser encontrada em baço, coração, rins, fígado, bexiga, pulmão, intestinos, pele de diversas regiões do corpo, subcutâneo e cavidade oral (FLORES et al., 2012; GÖRITZ et al., 2013; YAMAMOTO et al., 2013; SOARES et al., 2017). Macroscopicamente, o hemangiossarcoma cutâneo ou subcutâneo se apresenta como uma massa única, bem delimitada, de coloração vermelha a preta, consistência macia a firme, fluindo sangue ao corte. Microscopicamente, as células neoplásicas podem variar de formato fusiforme a ovalado, formando fissuras vasculares (HENDRICK, 2017).

Os quimiodectomas ou paragangliomas são neoplasias de quimiorreceptores que ocorrem na base do coração, e ao contrário das neoplasias primárias do coração, que são incomuns, podem ocorrer em cães e raramente em gatos e bovinos. Esses receptores detectam alteração de $\mathrm{pH}$, conteúdo de dióxido de carbono e oxigênio e auxiliam na regulação da respiração e circulação. Embora os órgãos quimiorreceptores estejam distribuídos em diversas partes do organismo, as neoplasias nos animais domésticos se originam dos corpos aórticos e carotídeos, sendo as primeiras mais frequentes (CAPEN, 2002). Macroscopicamente, os tumores do corpo aórtico evoluem para grandes massas, de consistência firme, que envolvem e comprimem grandes vasos e átrios. Microscopicamente, as células neoplásicas são poliédricas, com citoplasma vacuolizado e sustentadas por fino estroma de tecido conjuntivo (VLEET; FERRANS, 2009). Cães de raças braquicefálicas, como Boxer, são predispostos a essa neoplasia (CAPEN, 2002). Objetivou-se, com esse estudo, relatar o caso de ocorrência simultânea de hemangiossarcoma e quimiodectoma em uma cadela de raça Boxer.

\section{RELATO DE CASO}

Uma cadela, 13 anos, raça boxer, foi enviada ao setor de Patologia Animal da Universidade Federal de Uberlândia - MG para realização do exame necroscópico. Ante mortem, segundo dados obtidos na ficha clínica da paciente, foi observada massa em região de osso frontal, com crescimento progressivo, próxima ao olho direito com lacrimejamento desse e o animal apresentava tremores, incoordenação, fraqueza e perda de peso progressiva. Diante do quadro clínico do animal, o proprietário optou por eutanásia.

Ao exame de necroscopia, observou-se massa, de consistência macia, medindo aproximadamente $7,0 \mathrm{~cm}$ de diâmetro, em subcutâneo, na região de osso frontal, próxima ao globo ocular direito, apresentando, ao corte, material de aspecto gelatinoso e coloração vermelho-vinho e ausência de segmento de osso na região da massa (figura 1). Em corpo aórtico notou-se massa de aproximadamente $4,0 \mathrm{~cm}$, consistência firme, coloração esbranquiçada (figura 2). Logo após a necropsia, foi realizado radiografia do crânio onde constatou-se lise óssea por parte da massa (figura 3). Coletou-se fragmentos da massa na cabeça e massa no corpo aórtico para histopatológico, os quais foram processados rotineiramente para coloração de hematoxilina e eosina. 


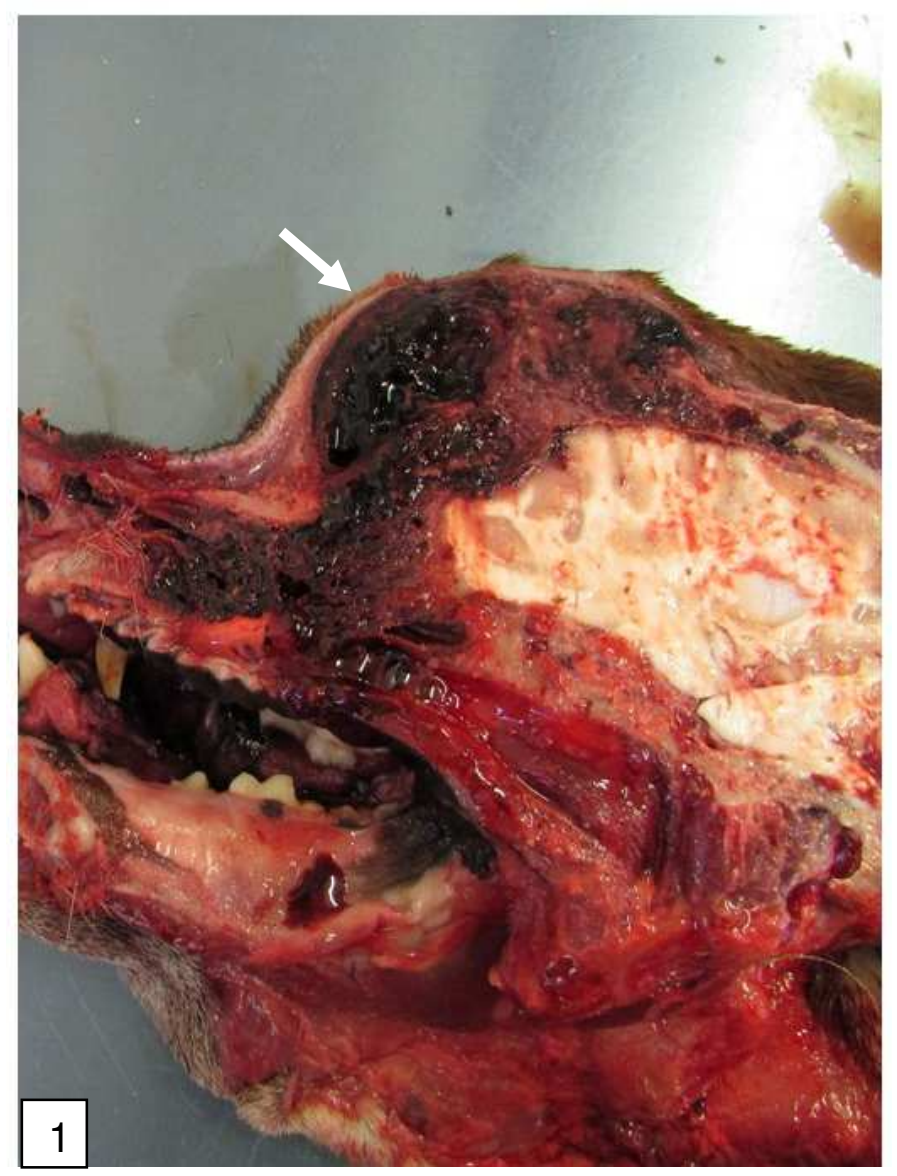

FIGURA 1: Cadela, Boxer, 13 anos. Corte sagital da cabeça evidenciando massa em região de osso frontal (seta). Fonte: arquivo Laboratório de Patologia Animal - UFU.

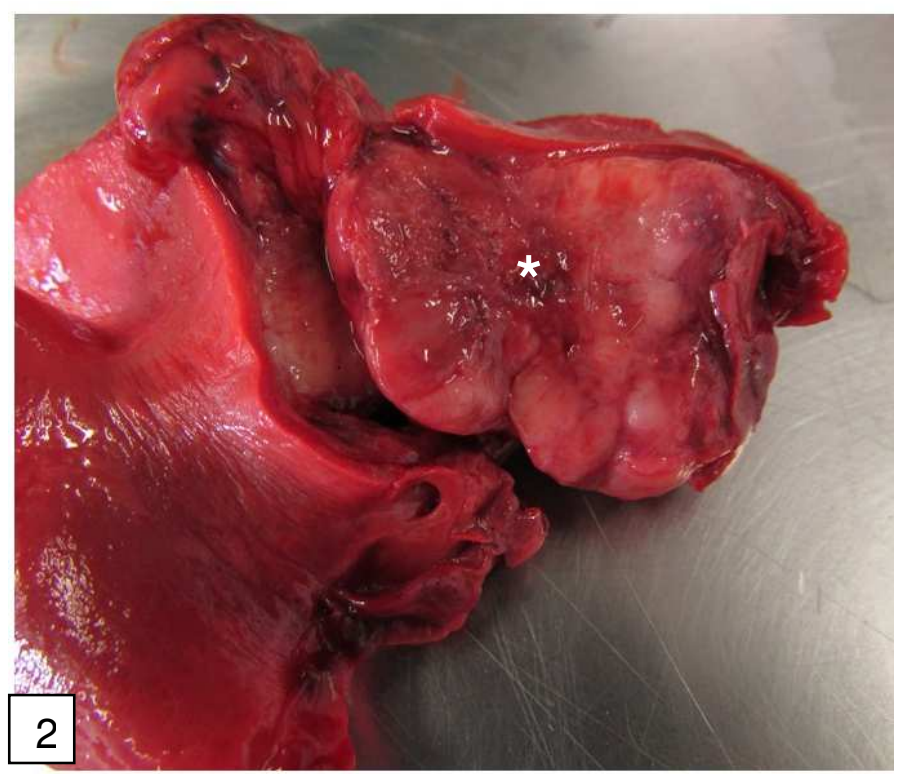

FIGURA 2: Cadela, Boxer, treze anos. Massa em aorta ascendente $\left(^{*}\right)$. Fonte: arquivo Laboratório de Patologia Animal - UFU. 


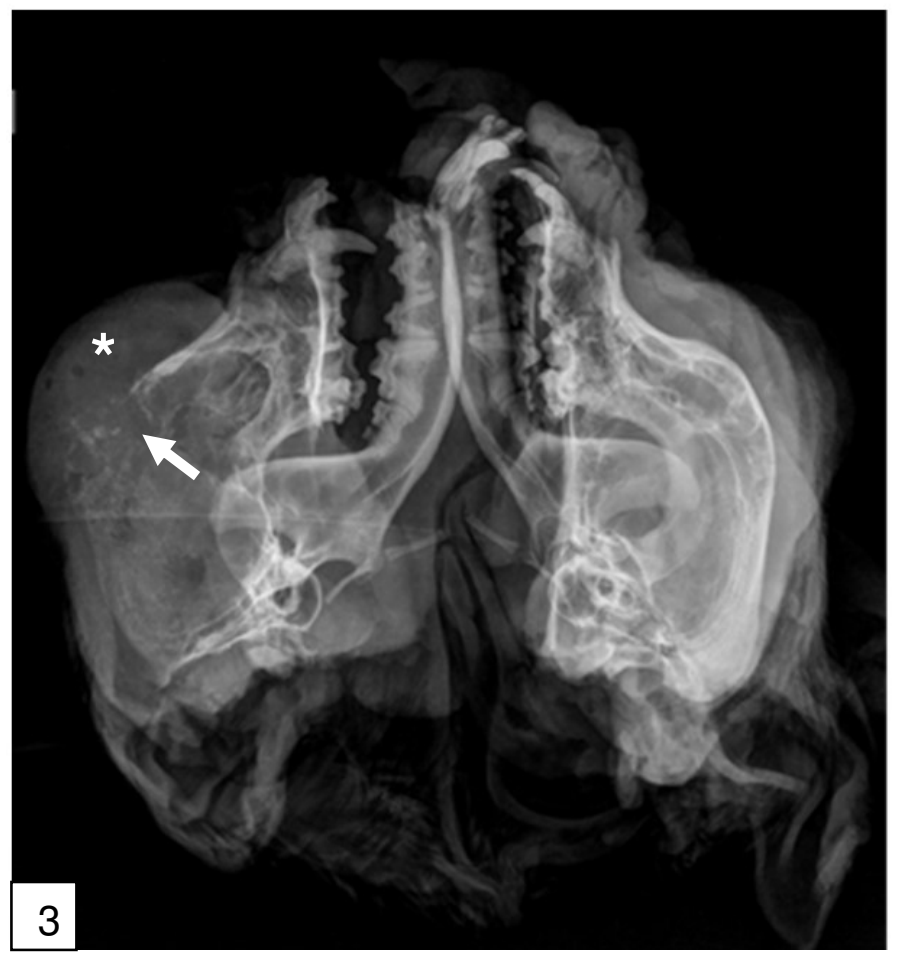

FIGURA 3: Cadela, Boxer, treze anos. Radiografia de crânio mostrando osteólise em osso frontal (seta). Presença de massa em região de osso frontal $\left({ }^{*}\right)$. Fonte: arquivo Laboratório de Patologia Animal - UFU.

\section{RESULTADOS E DISCUSSÃO}

A cadela deste relato era da raça Boxer e apresentava, ao exame histopatológico da massa na cabeça, neoformação vascular, com endotélio pouco diferenciado, formando fendas, que estavam preenchidas por hemácias, levando ao diagnóstico de hemangiossarcoma subcutâneo (figura 4). Dados estes que corroboram com Santos e Alessi (2017) e Goritz et al., (2013), que afirmam que o hemangiossarcoma pode se originar em qualquer lugar que seja vascularizado, fato esse que inclui pele e subcutâneo. Cães são acometidos com frequência, e a raça Boxer está entre as mais acometidas por essa neoplasia (FLORES et al., 2012; SILVEIRA et al., 2012; SOARES et al., 2017) concordando com o descrito no presente relato.

Apesar de descrito como menos agressivo que a forma visceral (HENDRICK, 2017), o hemangiossarcoma em questão apresentou comportamento biológico invasivo, causando osteólise do osso frontal, atingindo encéfalo e provocando sinais neurológicos no animal. Porém, metástases não foram encontradas. Tumores do corpo aórtico são encontrados em cães e incomuns em outras espécies (SANTOS; ALESSI, 2017). Já foram relatadas algumas raças com essa neoplasia: Fila Brasileiro (GUNDIM et al., 2017), Weimaraner (ARAÚJO et al., 2017), Golden Retriever (ZANONI, 2016), Sem raça definida (BERTOLO, 2017) e principalmente em Boxer (MESQUITA 2012; SANTOS; ALESSI, 2017). Em geral, a média de idade dos animais acometidos por quimiodectoma varia entre oito e dez anos (MESQUITA, 2012; ZANONI, 2016; BERTOLO, 2017; ARAÚJO et al., 2017). Os sinais clínicos estão associados ao tamanho do tumor, que pode comprimir a parede vascular ou a base do coração (SANTOS; ALESSI, 2017), podendo variar entre sopro cardíaco, 
apatia, tosse, dispneia, mucosas hipocoradas, taquipinéia, dentre outros que podem ser inespecíficos (SALOMÃO et al., 2012; MESQUITA, 2012; ARAÚJO et al., 2017).

Macroscopicamente, a massa em corpo aórtico apresentava consistência firme e coloração esbranquiçada e microscopicamente observou-se aglomerados de células de formato poliédrico, citoplasma vacuolizado, núcleo redondo a oval, de localização central, separados por fino estroma de tecido conjuntivo (figura 5), de acordo com descrito por Santos e Alessi (2017), levando ao diagnóstico de quimiodectoma. Além disso, a raça e idade da cadela são compatíveis com relatos anteriores, no entanto, o animal não apresentava sintomatologia, além sinais neurológicos.

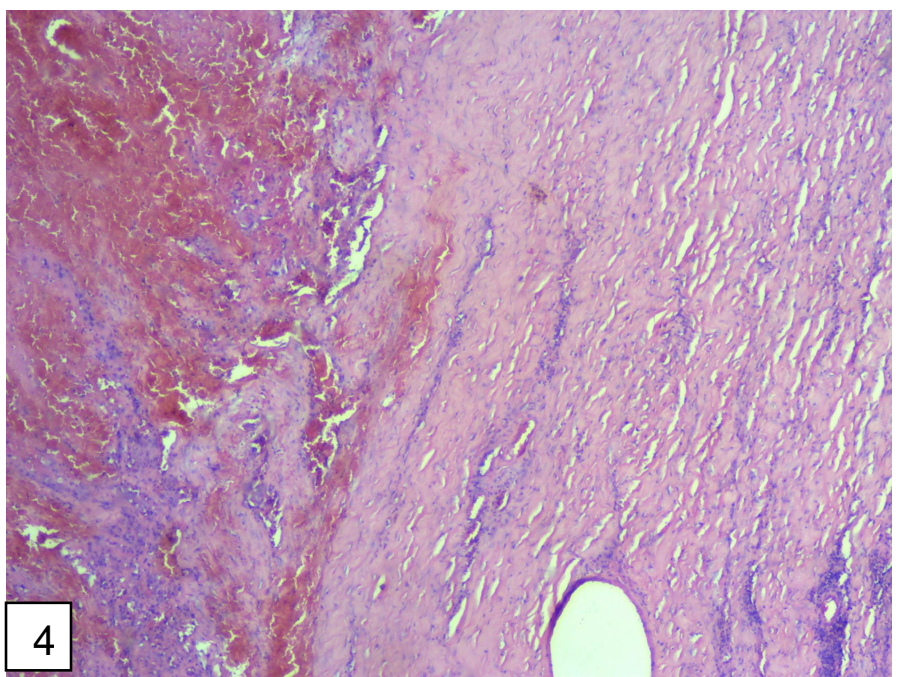

FIGURA 4: Cadela, Boxer, treze anos. Notar neoformação vascular, em formato de fendas, preenchidas por hemácias. HE. Aumento 4x. Fonte: arquivo Laboratório de Patologia Animal UFU.

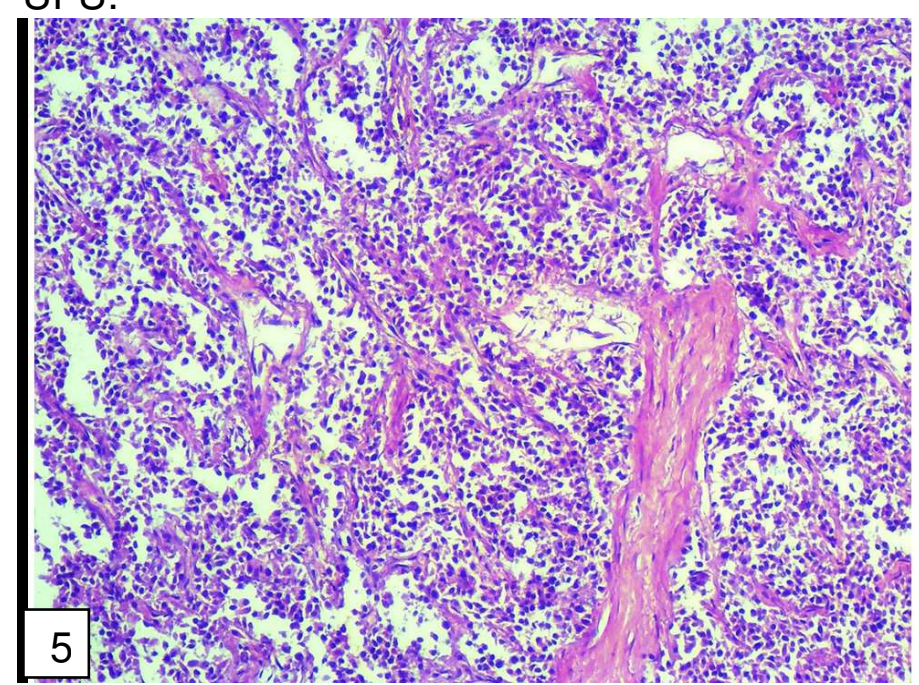

FIGURA 5: Cadela, Boxer, treze anos. Notar aglomerados de células de formato poliédrico, citoplasma vacuolizado, núcleo redondo a oval, de localização central, separados por fino estroma de tecido conjuntivo. HE. Aumento 4x. Fonte: arquivo Laboratório de Patologia Animal UFU. 


\section{CONCLUSÃO}

Com o presente relato, conclui-se que neoplasias de classificações histológicas diferentes e em sítios anatômicos distintos podem ocorrer simultaneamente em um mesmo animal. O hemangiossarcoma descrito apresentou comportamento extremamente agressivo, localmente, ao ponto de causar osteólise de osso frontal devido ao seu crescimento e provocar sinais neurológicos ao animal. Concomitantemente, ao exame necroscópico, encontrou-se um quimiodectoma de arco aórtico, o qual não induzia nenhuma sintomatologia ao paciente, sendo neste caso um achado acidental, constatando-se que essa neoplasia pode ser silenciosa e não provocar sinais clínicos. Faz-se importante a realização de exames complementares, principalmente de imagem, para pesquisa de mais focos tumorais, visto que neoplasias podem não provocar sintomatologia clínica, a exemplo desse estudo.

\section{REFERÊNCIAS}

ARAÚJO, G. D., WILSON, T. M., BERNARDO, D. M., CAMPOS, M. T. G., ARAÚJO, D. N. Quimiodectoma maligno em um cão Weimaraner: Relato de caso. Pubvet, v.11, n.7, p. 701-704, 2017. Disponível em: < http://www.pubvet.com.br/artigo/3930/quimiodectoma-maligno-em-um-catildeoweimaraner-relato-de-caso >. Doi: http://dx.doi.org/10.22256/pubvet.v11n7.701-704

BERTOLO, P. H. L., AGUIRRA, L. R. V. M., MONGER, S. D. G. B., CARDOSO, A. M. C., VASCONCELOS, R. O., PEREIRA, W. L. A. Paraganglioma de corpo aórtico em cão. Acta Scientiae Veterinariae, v. 45, p. 1-4, 2017. Disponível em: <http://revistas.bvs-vet.org.br/actascivet/article/view/37524>. ISSN 1679-9216

CAPEN, C.C. Tumors of the Endocrine Glands. In: MEUTEN, D. Tumors in domestic animals, 4 ed. Ames: lowa State, 2002. p. 601-695.

CHIKAZAWA, S., HORI, Y., HOSHI, F., KANAI, K., ITO, N., \& HIGUCHI, S. Hyperferritinemia in dogs with splenic hemangiosarcoma. Journal of Veterinary Medical Science, v. 75 , n. 11, p. 1515-1518, 2013. Disponível em: < https://www.jstage.jst.go.jp/article/jvms/75/11/75_13-0147/_article > Doi: http://doi.org/10.1292/jvms.13-0147

FLORES, M. M., PANZIERA, W., KOMMERS, G. D., IRIGOYEN, L. F., BARROS, C. S., FIGHERA, R. A. Aspectos epidemiológicos e anatomopatológicos do hemagiossarcoma em cães: 40 casos (1965-2012). Pesquisa Veterinária Brasileira vol.32 no.12 Rio de Janeiro dez. 2012. Disponível em: < http://www.scielo.br/scielo.php?script=sci_arttext\&pid=S0100-

736X2012001200017\&lng=pt\&tlng=pt $>$ Doi: http://dx.doi.org/10.1590/S0100736X2012001200017

GÖRITZ, M., MÜLLER, K., KRASTEL, D., STAUDACHER, G., SCHMIDT, P., KÜHN, M., NICKEL, R., SCHOON, H. A. Canine splenic haemangiosarcoma: influence of metastases, chemotherapy and growth pattern on post-splenectomy survival and expression of angiogenic factors. Journal of comparative pathology, v. 149, n. 1, p. 30-39, 2013. Disponível em: 
<http://www.sciencedirect.com/science/article/pii/S0021997512004227>

Doi: https://doi.org/10.1016/j.jcpa.2012.11.234

GUNDIM, L. F., MOREIRA, T. A., SOUZA, R. R., BANDARRA, M. B., MEDEIROS, A. A. Quimiodectoma em Cão - Relato de Caso. Enciclopédia Biosfera, v.11, n.22, p. 2015. http://www.conhecer.org.br/enciclop/2015c/agrarias/quimiodectoma.pdf >. Doi: http://dx.doi.org/10.18677/Enciclopedia_Biosfera_2015_218

HENDRICK, M. J. Mesenchymal Tumors of the Skin and Soft Tissues. In: D. J. Meuten (Ed.), Tumors in Domestic Animals, (5 th edit.), lowa State Press, Ames (2017), pp. 142-175.

LAMERATO-KOZICKI, A. R., HELM, K. M., JUBALA, C. M., CUTTER, G. C., MODIANO, J. F. Canine hemangiosarcoma originates from hematopoietic precursors with potential for endothelial differentiation. Experimental hematology, v. 34, n. 7, p. 870-878, 2006. Disponível em: < http://www.sciencedirect.com/science/article/pii/S0301472X06002621> Doi: http://dx.doi.org/10.1016/j.exphem.2006.04.013

MESQUiTA, L. P., ABREU, C. C., NOGUEIRA, C. I., WOUTERS, A. T., WOUTERS, F., JÚNIOR, P. S. B., MUZZI, R. A. L., VARASCHIN, M. S. Prevalência e aspectos anatomopatológicos das neoplasias primárias do coração, de tecidos da base do coração e metastáticas, em cães do Sul de Minas Gerais (1994-2009). Pesquisa Veterinária Brasileira, v. 32, n. 11, p. 1155-1163, 2012. Disponível em: <http://www.pvb.com.br/pdf_artigos/01-11-2012_11-

35Vet\%201301_2788\%20PA.pdf>. DOI: http://dx.doi.org/10.1590/S0100736X2012001100014

SALOMÃO, M. C., MATTOS, A. S. D., LUCENA, A. R., LEITE, J. D. S., MELLO, M. F. V. D., FERREIRA, A. M. R. Tumor de arco aórtico em cão (Canis familiaris)- relato de caso. Veterinária e Zootecnia v.19, n.1-S.1, p.107-109, 2012. Disponível em: < http://www.fmvz.unesp.br/rvz/index.php/rvz/article/view/249/206>. ISSN 0102-5716

SANTOS, R. L., ALESSI, A. C. Patologia Veterinária. 2 ed. Rio de Janeiro: Rocca 2017. p.842.

SILVEIRA, M. F., DE OLIVEIRA GAMBA, C., GUIM, T. N., BONEL-RAPOSO, J., \& FERNANDES, C. G. Características Epidemiológicas De Sarcomas De Tecidosmoles Caninos E Felinos: Levantamento De 30 Anos. Revista Acadêmica: Ciência Animal, v. 10, n. 4, p. 361-365, 2012. Disponível em: < https://periodicos.pucpr.br/index.php/cienciaanimal/article/view/12314/11648>. Doi: 10.7213/academica.7742

SOARES, N. P., MEdeiRos, A. A., SzABÓ, M. P. J., GUIMARÃES, E. C., FERNANDES, L. G., DOS SANTOS, T. R. Hemangiomas e hemangiossarcomas em cães: estudo retrospectivo de 192 casos (2002-2014). Ciência Animal Brasileira, v. 
18, p. 01-10, 2017. Disponível em: < https://www.revistas.ufg.br/vet/article/view/e30889/23165 > Doi: http://dx.doi.org/10.1590/1089-6891v18e-30889

VLEET, J. F. V., FERRANS, V. J. Sistema Cardiovascular. In: McGAVIN, M.D; ZACHARY, J. F. Bases da Patologia em Veterinária. 4 ed. Rio de Janeiro: Elsevier, 2009. p. $559-612$.

YAMAMOTO, S., HOSHI, K., HIRAKAWA, A., CHIMURA, S., KOBAYASHI, M., MACHIDA, N. Epidemiological, clinical and pathological features of primary cardiac hemangiosarcoma in dogs: a review of 51 cases. Journal of Veterinary Medical Science, v. 75 , n. 11, p. 1433-1441, 2013. Disponível em: <https://www.jstage.jst.go.jp/article/jvms/75/11/75_13-0064/_article>. Doi: http://doi.org/10.1292/jvms.13-0064

ZANONI DS, FERIOLI RB, XAVIER JG, SEQUEIRA JL, AMORIM RL. Paraganglioma em cão associado à falência cardíaca: Relato de Caso. Veterinária e Zootecnia, v. 23, n. 1, p. 72-77, 2016. Disponível em: <http://www.fmvz.unesp.br/rvz/index.php/rvz/article/view/788>. $\quad$ ISSN 0102-5716 ISSN Eletrônico 2178-3764 